\title{
CXCR7 silencing inhibits the migration and invasion of human tumor endothelial cells derived from hepatocellular carcinoma by suppressing STAT3
}

\author{
YE WU $^{1,2 *}$, LEI TIAN ${ }^{3 *}$, YONGLE XU ${ }^{2}$, MINHONG ZHANG ${ }^{2}$, \\ SHENGQING XIANG $^{4}$, JIANGUO ZHAO ${ }^{1}$ and ZHENXIA WANG ${ }^{1}$
}

\author{
${ }^{1}$ Department of General Surgery, Affiliated Hospital of Inner Mongolia Medical University, Hohhot, \\ Inner Mongolia 010059; ${ }^{2}$ Department of Vascular Surgery, General Hospital of PLA, Beijing 100853; \\ Departments of ${ }^{3}$ Anesthesia and ${ }^{4}$ Pharmacy, General Hospital of The PLA Rocket Force, Beijing 100088, P.R. China
}

Received November 12, 2017; Accepted May 11, 2018

DOI: $10.3892 / \mathrm{mmr} .2018 .9114$

\begin{abstract}
C-X-C chemokine receptor type 7 (CXCR7) is reported to be overexpressed in tumor endothelial cells (TECs), which are the primary target cells of antivascular chemotherapy. However, the role of CXCR7 in TECs is not fully understood. In the present study, CXCR7 expression was inhibited in TECs derived from hepatocellular carcinoma (HCC) using short hairpin (sh)RNA plasmids to investigate the role of CXCR7 in the regulation of migration and invasion of TECs as well as its underlying mechanisms. The data showed that the downregulation of CXCR7 significantly inhibited the migration and invasion of TECs. Further study showed that silencing CXCR7 resulted in decreased phosphorylated signal transducer and activator of transcription 3 (STAT3) at Tyr705 and its downstream target genes in TECs, including matrix metalloproteinase-2 (MMP2) and vascular endothelial growth factor (VEGF). However, restoring STAT3 phosphorylation abolished the CXCR7-shRNA-induced decrease in TECs migration and invasion, as well as the downregulation of MMP2 and VEGF in TECs. These findings indicate that CXCR7 may regulate the migration and invasion of TECs derived from HCC via the STAT3 signaling pathway and that CXCR7 could be a potential target for the antivascular therapy of HCC.
\end{abstract}

Correspondence to: Professor Zhenxia Wang, Department of General Surgery, Affiliated Hospital of Inner Mongolia Medical University, 1 TongDao North Street, Hohhot, Inner Mongolia 010059, P.R. China

E-mail: wzhenxia@163.com

*Contributed equally

Key words: tumor endothelial cells, C-X-C chemokine receptor type 7, signal transducer and activator of transcription 3, migration, invasion

\section{Introduction}

Hepatocellular carcinoma (HCC) is one of the most common and lethal malignancies worldwide, especially in Asia and Africa (1). The main cause of cancer-related deaths in HCC patients is tumor metastasis, which relies on angiogenesis (2). It has been proposed that the metastatic activity of tumors is related to whether they have acquired an angiogenic phenotype (the ability to recruit vasculature), which enables them to obtain adequate nutrition and oxygen by angiogenesis $(3,4)$. As the pivotal component of tumor blood vessels, tumor endothelial cells (TECs) perform a very crucial role during tumor angiogenesis. The migratory and invasive capacities of TECs enable them to degrade the surrounding ECM and migrate towards pro-angiogenic cues to form neovessels. Thus, understanding the mechanisms that regulate the migration and invasion of TECs is very important for the antiangiogenic therapy of cancer.

$\mathrm{C}-\mathrm{X}-\mathrm{C}$ chemokine receptor type 7 (CXCR7) is a $\mathrm{G}$-protein-coupled chemokine receptor, and its ligands are CXCL12 and CXCL11. Reportedly, CXCR7 was upregulated in cancer cells and mediates a broad range of cellular activities, including proliferation, survival, and metastasis, by binding to CXCL12 $(5,6)$. A recent study showed that CXCR7 was overexpressed in TECs derived from renal cell carcinoma (7). Further study indicated that the expression of CXCR7 in TECs derived from renal cell carcinoma is important for the migration and tube formation of TECs and for tumor angiogenesis in vivo (8). Moreover, upregulating CXCR7 in human $\mathrm{HCC}$ was found to increase the angiogenic capacity of human umbilical vein endothelial cells (HUVECs) in in vitro tube formation assays (9). This indicated that CXCR7 might be associated with the functional regulation of TECs in HCC. However, the role of $\mathrm{CXCR} 7$ in regulating the invasion and migration of TECs in HCC as well as the underlying mechanisms have not been adequately described. Therefore, in the current study, we investigated the effect of CXCR7 silencing on the migration and invasion of TECs derived from HCC in vitro and further explored the mechanisms involved in CXCR7-regulated migration and invasion of TECs derived from HCC. Our data 
demonstrate that $\mathrm{CXCR} 7$ is an important molecule in regulating the migration and invasion of TECs derived from $\mathrm{HCC}$ by triggering the signal transducer and activator of transcription 3 (STAT3) pathway.

\section{Materials and methods}

Cell lines. TECs from HCC were purchased from Sixin Biotech (Shanghai, China; cat. no. TCHU82), routinely maintained in DMEM supplemented with 10\% FBS and penicillin-streptomycin solution, and cultured at $37^{\circ} \mathrm{C}$ in a humid atmosphere with $5 \% \mathrm{CO}_{2}$.

CXCR7 knockdown and STAT3 over expression. The TEC cells were seeded in a six-well plate at $1.0 \times 10^{6}$ cells/well. When cells were $70-90 \%$ confluent, they were transfected with a short hairpin (sh)RNA plasmid targeting CXCR7 (CXCR7-shRNA) or empty shRNA plasmid (NC-shRNA) (both from Santa Cruz Biotechnology, Inc., Dallas, TX, USA) using Lipofectamine 3000 reagent (Invitrogen; Thermo Fisher Scientific, Inc., Waltham, MA, USA) according to the manufacturer's protocol. At $24 \mathrm{~h}$ after transfection, the cells were cultured in medium containing $2 \mu \mathrm{g} / \mathrm{ml}$ puromycin for 7 days to select stably transfected TECs. The expression of CXCR7 in the surviving clones was measured using reverse transcription-quantitative polymerase chain reaction (RT-qPCR) and immunoblotting.

For STAT3 overexpression, the pcDNA3.0 plasmid expressing activated STAT3 (pcDNA3.0-STAT3) or empty pcDNA3.0 plasmid (pcDNA3.0) (a kind gift from Dr. Fengsheng Li) was transiently transfected into TECs with a stable downregulation of CXCR7 using Lipofectamine 3000 reagent (Invitrogen; Thermo Fisher Scientific, Inc.) according to the manufacturer's protocol. At $48 \mathrm{~h}$ after transfection, the cells were harvested for subsequent experiments.

$R T-q P C R$. Total RNA was extracted from TECs transfected with or without pcDNA3.0-STAT3, pcDNA3.0, CXCR7-shRNA or NC-shRNA using TRIzol reagent (Invitrogen; Thermo Fisher Scientific, Inc.). First-strand cDNA was then generated using ALL-in-One ${ }^{\mathrm{TM}}$ First-Stand cDNA Synthesis kit (GeneCopoeia, Rockville, MD, USA) according to the manufacturer's protocol. RT-qPCR was carried out using Talent qPCR PreMix (SYBR-Green) kit (Tiangen Biotech, Beijing, China) according to the manufacturer's protocol. The primer sequences used in the current study are shown in Table I. The relative level of target mRNA was determined by the $2^{-\Delta \Delta \mathrm{Cq}}$ method.

Immunoblotting. Total protein from TECs was collected by using a lysis buffer (1\% Triton X-100, $150 \mathrm{mmol} / \mathrm{l} \mathrm{NaCl}$, $50 \mathrm{mmol} / 1$ Tris, $\mathrm{pH}$ 8.0, $1 \mathrm{mmol} / 1$ EDTA, $10 \mathrm{mg} / \mathrm{l}$ henylmethylsulfonyl fluoride). The protein concentration was determined by the BCA Protein Assay kit (Tiangen Biotech) according to the manufacturer's protocol. Then, $50 \mu \mathrm{g}$ protein was separated by $10 \%$ SDS-PAGE and transferred onto a PVDF membrane. After blocking with a 5\% BSA in PBS, the membrane was incubated with anti-CXCR7 antibody $(1: 1,000$; Abgent, San Diego, CA, USA), anti-STAT3 (phospho Y705) antibody (1:1,000; Cell Signaling Technology, Inc., Danvers,
MA, USA), anti-matrix metalloproteinase-2 (MMP2) antibody (1:1,000; Santa Cruz Biotechnology, Inc., Dallas, TX, USA), anti-vascular endothelial growth factor (VEGF) antibody (1:1,000; Abgent) or anti-Tubulin antibody (1:5,000, YTHX Biotechnology Co. Ltd, Beijing, China) overnight at $4^{\circ} \mathrm{C}$. After washing with Tris-buffered saline $(\mathrm{pH}$ 7.2) containing $0.05 \%$ Tween-20, the membrane was incubated with the secondary antibody and subsequently visualized using the ECL system.

Cell migration assay. The migration of TECs was measured by both transwell migration assay and wound healing assay, as described in previous studies $(10,11)$. For the transwell migration assay, briefly, $5 \times 10^{4}$ TECs in $200 \mu$ of serum-free DMEM was added to the top compartment of the transwell insert-setup (Corning Incorporated, Corning, NY, USA). Then, $500 \mu \mathrm{l}$ of DMEM medium supplemented with $10 \%$ FBS was added to the bottom chamber. Following incubation for $12 \mathrm{~h}$ at $37^{\circ} \mathrm{C}$ in a humid atmosphere with $5 \% \mathrm{CO}_{2}$, the cells on the lower surface of the membrane were fixed with $100 \%$ methanol and stained with Giemsa. Migrated cells were counted and expressed as an average number of cells/microscopic field. For the wound healing assay, TECs with normal or downregulated CXCR7 expression were seeded into 6-well plates and grown to confluence. After washing with serum-free medium, the confluent cells were wounded with a $200 \mu$ l pipette tip followed by washing with serum-free medium to remove the non-adherent cells. The wounded monolayers were subsequently incubated with serum-free DMEM at $37^{\circ} \mathrm{C}$ in a humid atmosphere with $5 \% \mathrm{CO}_{2}$ for $24 \mathrm{~h}$. The cells were observed by phase-contrast microscopy, and images were captured (magnification, x100).

Invasion assay. The invasive capacity of TEC cells was measured using a transwell invasion assay. Briefly, $0.1 \mathrm{ml}$ Matrigel (BD Biosciences, Franklin Lakes, NJ, USA) diluted by serum-free DMEM was added into the upper chamber. After the Matrigel proteins polymerized, $5 \times 10^{4} \mathrm{TECs}$ that had been grown in serum-free DMEM medium for $24 \mathrm{~h}$ and suspended in $200 \mu \mathrm{l}$ of FBS-free DMEM were added to the upper chamber. Then, $500 \mu 1$ of DMEM with $10 \%$ FBS was added to the lower chamber. The cell invasion chambers were incubated for $20 \mathrm{~h}$ at $37^{\circ} \mathrm{C}$ in a humid atmosphere with $5 \% \mathrm{CO}_{2}$. The cells on the lower surface of the membrane were fixed, stained and counted as described for the cell migration assay.

Statistical analysis. The experimental data are presented as the mean \pm standard deviation. One-way ANOVA followed by Student-Newman-Keuls test was performed to analyze the differences among groups using the statistical software SPSS 19.0 (SPSS, Inc., Chicago, IL, USA). P $<0.05$ was considered to indicate a statistically significant difference.

\section{Results}

The generation of TECs with the stable downregulation of $C X C R 7$. After selection with puromycin, the expression of CXCR7 in the surviving TEC clones was detected by RT-qPCR and immunoblotting. As shown in Fig. 1A, the transfection with NC-shRNA did not change the expression of CXCR7 mRNA in TECS compared to the untreated TECs. In 
Table I. Primer sequences.

Gene

Forward sequence (5'-3')

Reverse sequence (5'-3')

CXCR7

MMP2

VEGF

STAT3

$\beta$-actin
CACTGCTACATCTTGAACCT

ATGCCGTCGTGGACCTGC

GCACCCATGGCAGAAGGAGGAG

ATCACGCCTTCTACAGACTGC

ATTGCCGACAGGATGCAGAAG
GTTGATGGAGAAGATGAGGTGT

TGCTTCCAAACTTCACGCTCTT

GTGCTGACGCTAACTGACC

CATCCTGGAGATTCTCTACCACT

AGAAGCATTTGCGGTGGACG

CXCR7; C-X-C chemokine receptor type 7; MMP, matrix metalloproteinase; VEGF, vascular endothelial growth factor; STAT3, signal transducer and activator of transcription 3 .

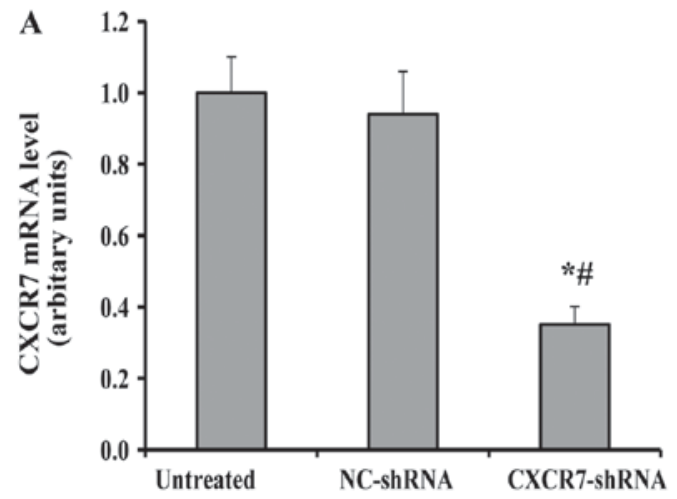

B

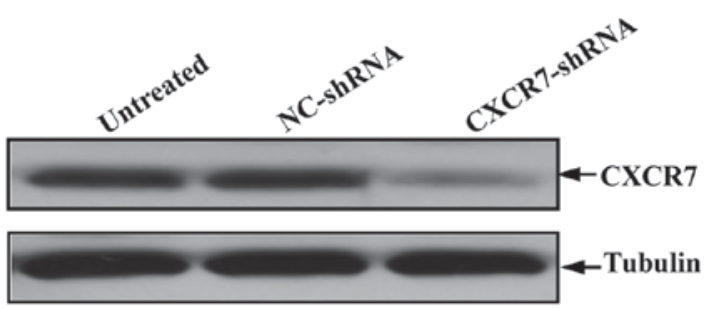

Figure 1. Transfection with CXCR7-shRNA significantly inhibits the expression of CXCR7 in TECs. TECs were transfected with CXCR7-shRNA or NC-shRNA and subsequently selected by puromycin. The expression of CXCR7 on the surviving TEC clone was detected by (A) RT-qPCR and (B) immunoblotting. " $\mathrm{P}<0.05$ vs. Untreated; ${ }^{*} \mathrm{P}<0.05$ vs. NC-shRNA. CXCR7; C-X-C chemokine receptor type 7; TEC, tumor endothelial cell; shRNA, short hairpin RNA.

contrast, the level of CXCR7 mRNA in TECs transfected with CXCR7-shRNA was significantly decreased. More importantly, a similar expression pattern of CXCR7 protein was observed in the immunoblotting assay (Fig. 1B). These results indicated that appropriate control and CXCR7 knock-down TECs were generated.

CXCR7 silencing inhibits the migration and invasion of TECs. The migratory capacity of TECs with stable CXCR7 downregulation was investigated using a transwell migration assay. As shown in Fig. 2A and B, TECs with stable NC-shRNA expression showed a similar migratory ability to that of the untreated TECs, but the migratory capacity of TECs with stable down-regulation of CXCR7 was significantly impaired compared to both untreated TECs and NC-shRNA-treated TECs, which was further confirmed by the wound healing assay (Fig. 2C). The invasive ability of TECs transfected with CXCR7 shRNA was further examined by a transwell invasion assay. Similarly, the transfection of NC-shRNA did not affect the invasive ability of TECs, but the TECs transfected with CXCR7 shRNA showed significantly impaired invasion (Fig. 2D and E).

CXCR7 silencing suppresses the STAT3 pathway in TECs. Previous studies have suggested that CXCR7 can trigger STAT3 expression, which has been found to contribute to cell migration, invasion, and tumor metastasis by transcriptionally regulating the expression of genes such as MMP2 and
VEGF (12-14). We therefore detected the expression of total and phosphorylated STAT3 in the TECs with stable CXCR7 downregulation. As shown in Fig. 3A, the inhibition of CXCR7 did not change the level of total STAT3 in TECs compared to untreated or NC-shRNA-transfected TECs. However, phosphorylated STAT3 was significantly decreased after CXCR7 silencing.

The expression of MMP2 and VEGF, which are 2 downstream genes of STAT3, in TECs was further investigated. As anticipated, the expression of MMP2 was significantly inhibited in TECs with stable CXCR7 downregulation at both the mRNA and protein levels compared to untreated or NC-shRNA-transfected TECs (Fig. 3B and C). Similarly, the downregulation of CXCR7 resulted in a significant decrease in the expression of VEGF mRNA and protein (Fig. 3C and D). These results indicated that the inhibition of CXCR7 suppressed the STAT3 pathway in TECs.

CXCR7 silencing suppresses the STAT3 pathway and decreases migration and invasion of TECs. We firstly examined the role of STAT3 in regulating migration and invasion of TECs. The TECs were transfected with or without pcDNA3.0-STAT3 of pcDNA3.0, and subsequently subjected to RT-qPCR, migration assay or invasion assay. As shown in Fig. 4A, the expression of STAT3 in TECs was significantly up regulated by transfection with pcDNA3.0-STAT3, compared to both pcDNA3.0 and untreated control; while treatment with pcDNA3.0 did not affect the expression of 

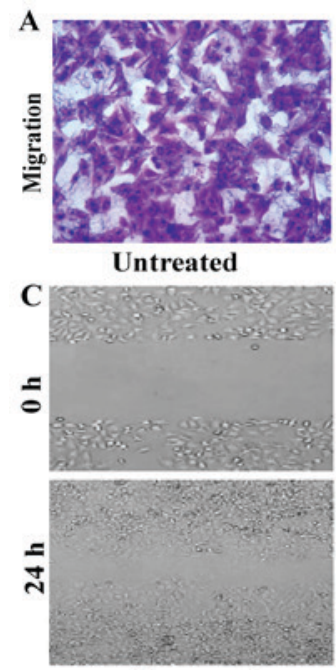

Untreated

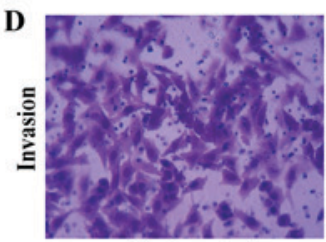

Untreated

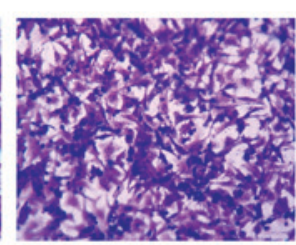

NC-shRNA

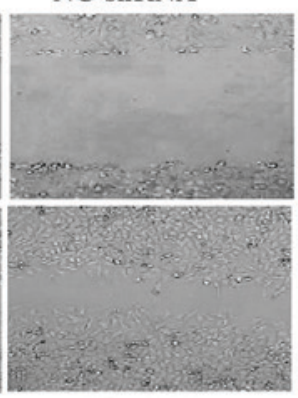

NC-shRNA

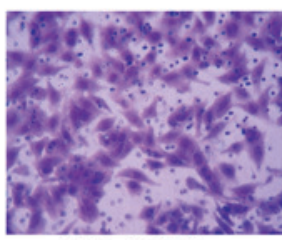

NC-shRNA

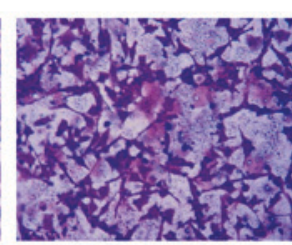

CXCR7-shRNA

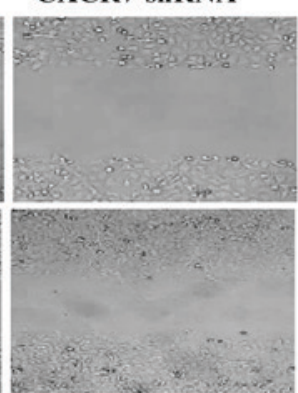

CXCR7-shRNA

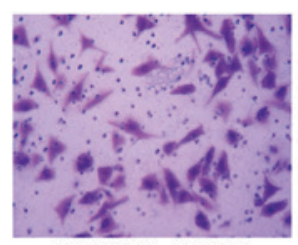

CXCR7-shRNA
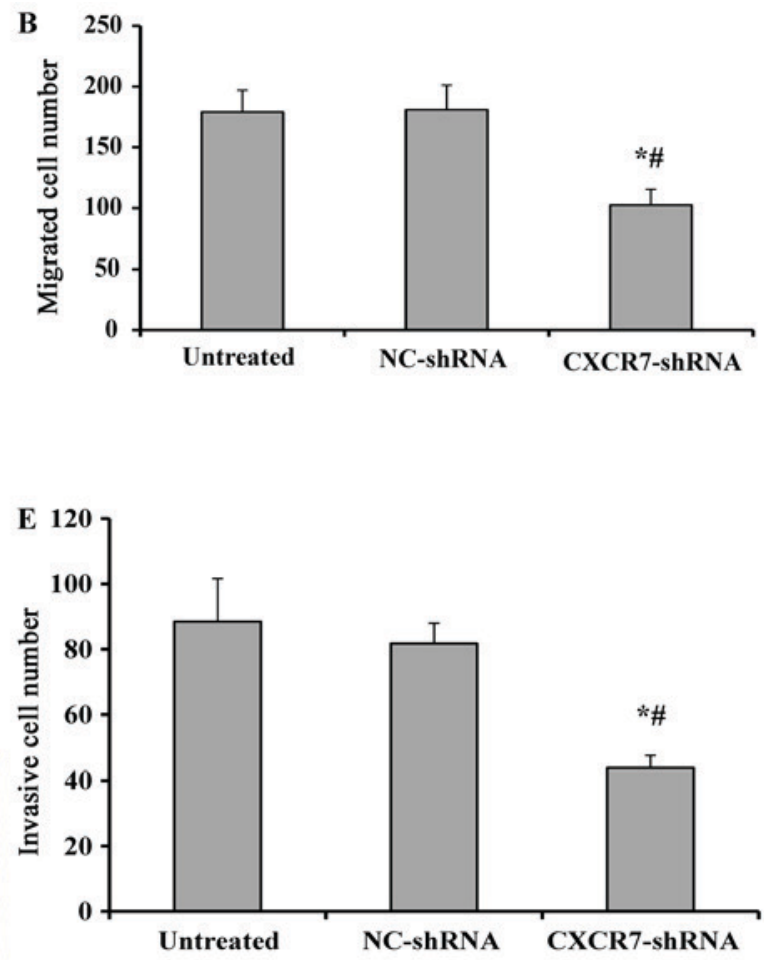

Figure 2. (A-E) Inhibition of CXCR7 significantly suppresses the migration and invasion of TECs. TECs with or without stable CXCR7 downregulation were subjected to a (A) Transwell migration assay (magnification, x200), (C) wound healing assay (magnification, x200) or (D) Transwell invasion assay (magnification, $\mathrm{x} 200)$. Quantification of the migrated cells in the (B) Transwell migration assay and the invasive cells in (E) Transwell invasion assay. ${ }^{*} \mathrm{P}<0.05$ vs. Untreated; ${ }^{\#} \mathrm{P}<0.05$ vs. NC-shRNA. CXCR7; C-X-C chemokine receptor type 7; TEC, tumor endothelial cell; shRNA, short hairpin RNA.

A

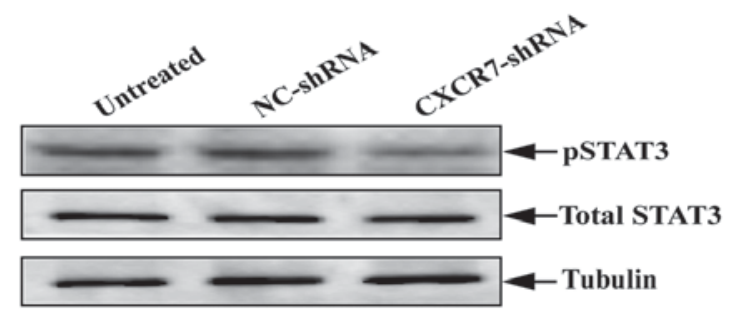

B

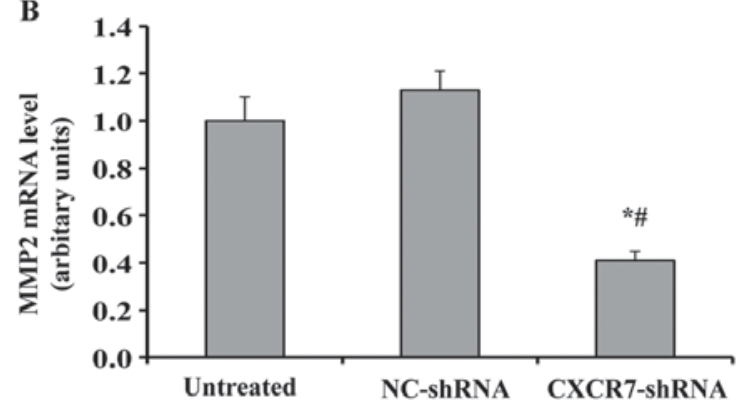

C

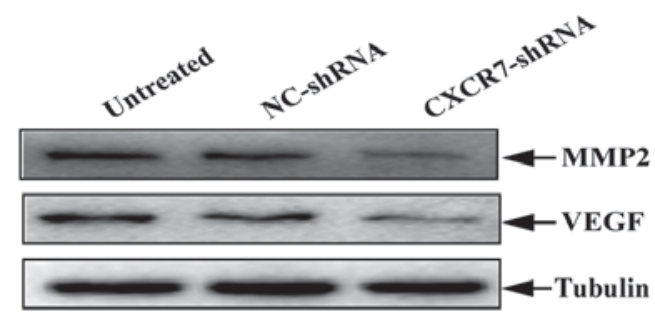

D

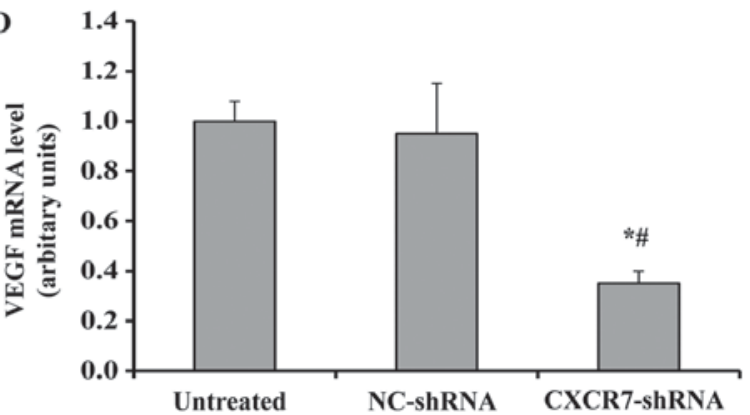

Figure 3. (A-D) Downregulation of CXCR7 inhibits the activity of STAT3 and target genes. Total protein from TECs with or without stable CXCR7 downregulation was collected, and the expression of (A) total and phosphorylated STAT3, (C) MMP2 and VEGF was detected by immunoblotting. The mRNA expression of (B) MMP2 and (D) VEGF in the total mRNA of TECs with or without stable CXCR7 downregulation was examined by RT-qPCR. "P<0.05 vs. Untreated; "P $<0.05$ vs. NC-shRNA. CXCR7; C-X-C chemokine receptor type 7; STAT3, signal transducer and activator of transcription 3; TEC, tumor endothelial cell; shRNA, short hairpin RNA.

STAT3 in TECs, compared to untreated control. Further study showed that transfection with pcDNA3.0-STAT3 increased the migration and invasion of TECs (Fig. 4B and C), which indicated that STAT3 was involved in the regulation of TEC migration and invasion.
To investigate whether the inhibition of the STAT3 pathway was necessary for the CXCR7 silencing-mediated decreases in TEC migration and invasion, the TECs with stable CXCR7 downregulation were transfected with pcDNA3.0-STAT3 or empty pcDNA3.0 plasmid. As shown 

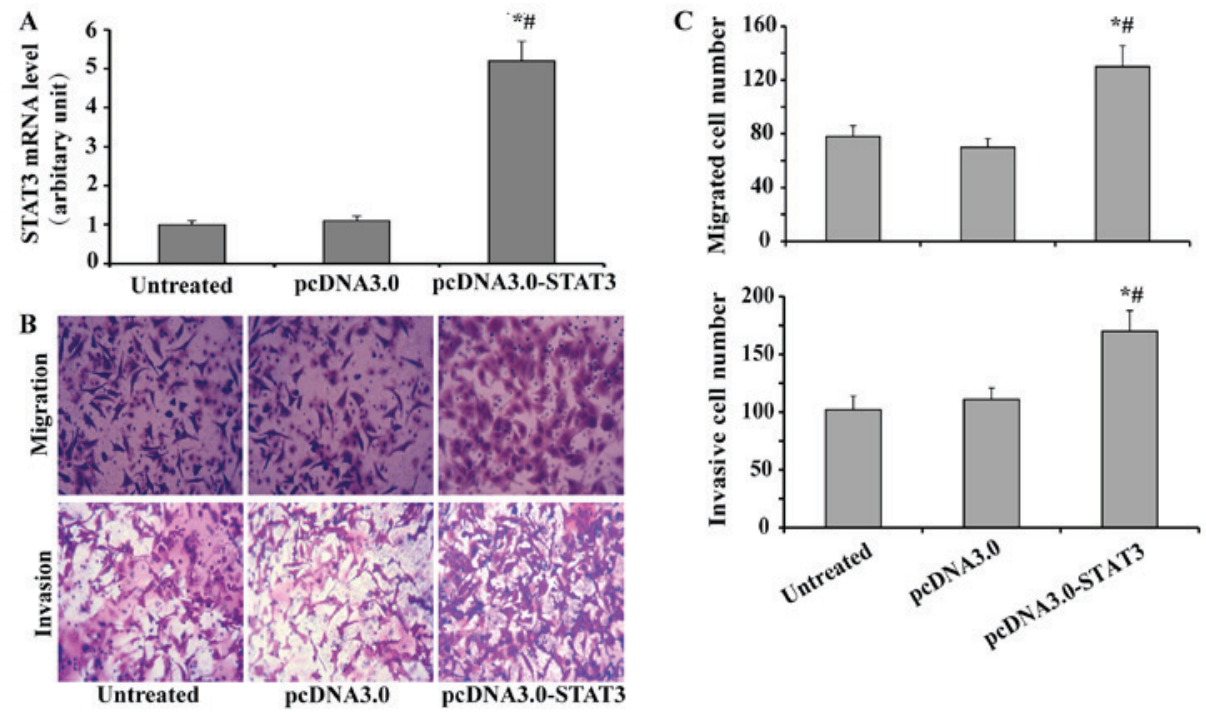

Figure 4. Overexpression of STAT3 enhances the migration and invasion of TECs. TECs were transfected with or without pcDNA3.0-STAT3 or pcDNA3.0. A total of $48 \mathrm{~h}$ after transfection, the cells were harvested and subjected to RT-qPCR, migration assay or invasion assay to detected the expression of (A) STAT3, (B) migratory and invasive capacity of cells (magnification, x200). Quantification of the migrated cells in the Transwell migration assay and the invasive cells in Transwell invasion assay $(C)$. ${ }^{*} \mathrm{P}<0.05$ vs. Untreated; ${ }^{*} \mathrm{P}<0.05$ vs. pcDNA3.0. STAT3, signal transducer and activator of transcription 3 ; TEC, tumor endothelial cell.

A

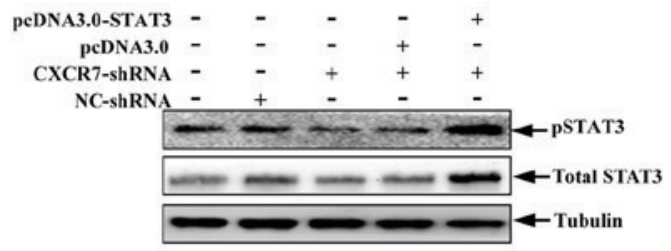

B

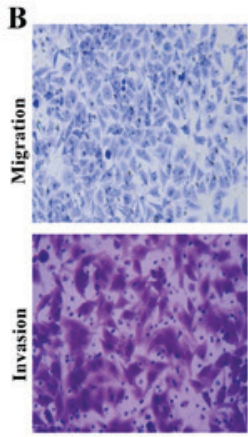

NC-shRNA

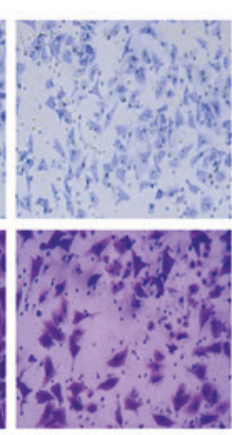

CXCR7-shRNA

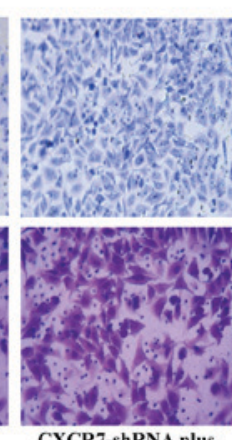
CXCR7-shRNA plus
peDNA3.0-STAT3
C
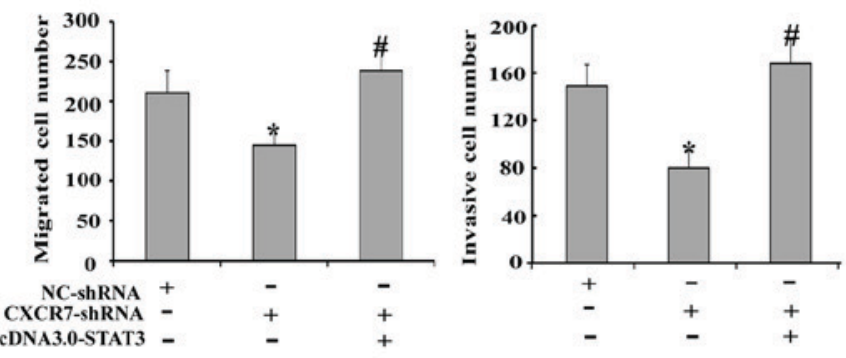

D
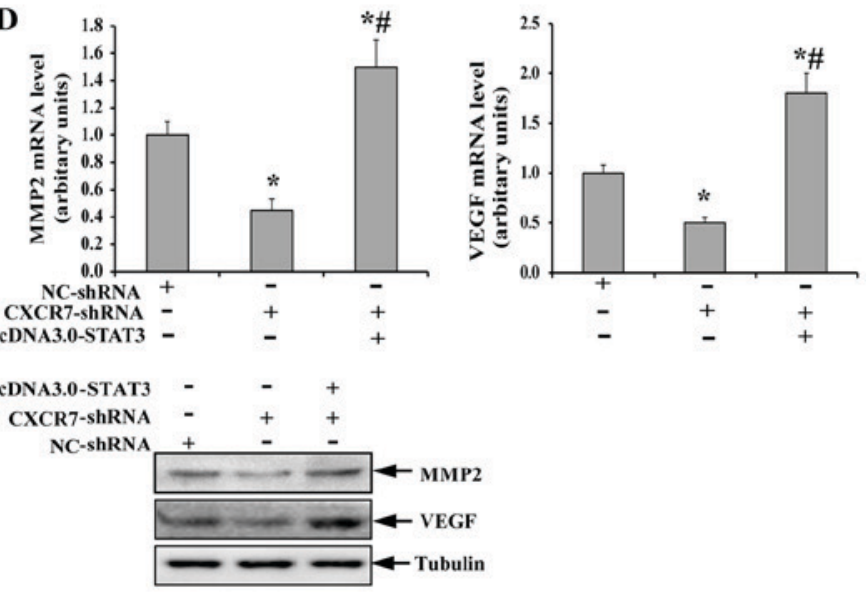

Figure 5. (A-D) Restoration of STAT3 activity abolishes the CXCR7 silencing-mediated decrease of TEC migration and invasion. TECs with stable CXCR7 downregulation were transfected with pcDNA3.0 or pcDNA3.0-STAT3. Following transfection for $48 \mathrm{~h}$, the cells were harvested and subjected to an immunoblotting assay to detect the expression of phosphorylated and total (A) STAT3, (D, lower panel) MMP2, and VEGF. (B) Transwell migration and invasion assays were used to examine the migratory and invasive capacity (magnification, x200), respectively. RT-qPCR was used to investigate the mRNA levels of MMP2 and VEGF (D, upper panel). (C) Quantification of the migrated cells in the Transwell migration assay and the invasive cells in Transwell invasion assay. "P<0.05 vs. NC-shRNA; "P<0.05 vs. CXCR7-shRNA. CXCR7, C-X-C chemokine receptor type 7; TEC, tumor endothelial cell; shRNA, short hairpin RNA; MMP, matrix metalloproteinase; VEGF, vascular endothelial growth factor.

in Fig. 5A, transfection with the empty pcDNA3.0 plasmid did not result in a significant change in phosphorylated STAT3 compared to TECs with stable CXCR7 downregulation. In contrast, treatment with pcDNA3.0-STAT3 significantly abolished the CXCR7-shRNA-induced downregulation of phosphorylated STAT3, accompanied by a significant 
increase in total STAT3. The levels of phosphorylated and total STAT3 in TECs treated with CXCR7-shRNA plus pcDNA3.0-STAT3 were even higher than those observed in untreated TECs. Further study showed that the decreased migratory and invasive capacities of TECs with stable CXCR7 downregulation were also abolished after the expression of phosphorylated STAT3 was restored (Fig. 5B and C). Compared to TECs transfected with NC-shRNA, TECs transfected with CXCR7-shRNA plus pcDNA3.0-STAT3 seemed to possess increased migratory and invasive capacities, but there was no significant difference in migratory or invasive capacity between the groups.

Finally, the expression of MMP2 and VEGF in TECs with decreased CXCR7 expression was also investigated after the restoration of phosphorylated STAT3 expression. As shown in Fig. 5D, the downregulation of MMP2 and VEGF in TECs induced by CXCR7 silencing was also abolished at both the mRNA and protein levels by transfection with pcDNA3.0-STAT3. Interestingly, the overexpression of constructively active STAT3 in TECs with CXCR7 silencing resulted in a significantly higher expression of MMP2 and VEGF than that of TECs with normal CXCR7 expression. These data indicated that the CXCR7 silencing mediated the decreased TEC migration and invasion abilities by suppressing the STAT3 pathway.

\section{Discussion}

One of key steps in the growth and metastasis of tumor is angiogenesis, which enables tumor cells to obtain sufficient nutrition and oxygen, and TECs are a critical component of angiogenesis, which makes them a major target of anti-angiogenic therapies (15). Understanding the mechanisms underlying various biologic behaviors of TECs, such as cell migration and invasion, is helpful for developing novel anti-angiogenic agents. The current study reveals that CXCR7 is involved in the migration and invasion of TECs derived from HCC. Further study showed that silencing CXCR7 resulted in a decrease in both phosphorylated STAT3 at Tyr705 and its downstream genes, MMP2 and VEGF, in TECs. Restoring STAT3 phosphorylation abolished the CXCR7-shRNA-mediated decrease of TEC migration and invasion and restored the expression of MMP2 and VEGF.

CXCR4, the 'canonical' receptor of CXCL12, has been demonstrated to promote tumor growth and metastasis in various tumor types by binding to CXCL12 $(16,17)$. CXCR7 has been recently identified as a second receptor for CXCL12 (18). It has been confirmed that CXCR7 is elevated in both malignant cells and tumor-associated blood vessels in human breast and lung cancer tissue (18). Although the overexpression of CXCR7 in tumor cells was shown to promote tumor growth, progression of metastasis, and epithelial-mesenchymal transition (EMT) $(18,19)$, the role of CXCR7 in TECs was not fully understood. Yamada et al first reported that CXCR7 was involved in migration, angiogenesis, and resistance to serum starvation in TECs derived from murine tumor A375SM xenografts (8). Consistently, our data showed that inhibiting CXCR7 in TECs derived from HCC suppressed cell migration and invasion in vitro. Because the migration and invasion of TECs are essential for angiogenesis in both primary and metastatic HCC lesions, this finding indicated that targeting TECs in HCC might be a potential strategy for treating HCC.

Various molecules have been reported to be downstream of CXCR7, including extracellular regulated protein kinases $1 / 2$ (ERK1/2) in epithelial ovarian carcinomas (20), mitogen-activated protein kinase (MAPK) in HCC (21), AKT in epithelial ovarian carcinomas and HCC $(9,20)$, and STAT3 in breast cancer (12). STAT3 belongs to the STAT protein family, whose role has been demonstrated to transmit various stimulatory signals from the cell membrane to the nucleus, where it transcriptionally regulates the expression of diverse target genes to response to the stimuli (22). STAT3 has been proven to promote the development and metastasis of various tumor types by elevating the expression of its target genes, such as VEGF, MMP2 and survivin (23-25). Wani et al (12) reported that CXCR7 expression enhanced the growth and metastasis of breast cancer by activating the STAT3/MMP2 pathway. In the current study, we revealed that inhibiting CXCR7 in TECs suppressed the activity of STAT3, followed by the downregulation of MMP2 and VEGF. This result indicated that STAT3 was one of downstream molecules of CXCR7 in TECs derived from HHC. The restoration of activated STAT3 in TECs abolished the CXCR7 inhibition-mediated decrease of cell migration and invasion as well as the downregulation of MMP2 and VEGF, indicating that CXCR7 regulates the migration and invasion of TECs via STAT3. Moreover, the overexpression of constitutively activated STAT3 resulted in significantly increased MMP2 and VEGF expression in TECs with CXCR7 silencing, which was accompanied by an enhanced migratory and invasive capacity of these cells. This was in line with the finding that TECs with CXCR7 silencing and STAT3 overexpression have a higher level of phosphorylated STAT3.

In conclusion, the current study demonstrates that CXCR7 plays an important role in regulating the migration and invasion of TECs derived from HCC. STAT3 is responsible for CXCR7-mediated migration and invasion in TECs derived from HCC. Targeting CXCR7 may be an effective antiangiogenic strategy for the treatment of HCC. Further in vivo studies are needed in future research.

\section{Acknowledgements}

The authors would like to thank Dr. Chenyi Guo from General Hospital of the PLA Rocket Force (Beijing, China) for helpful suggestions during statistical analysis.

\section{Funding}

This study was supported by the Key Project of The Affiliated Hospital of Inner Mongolia Medical University (grant no. NYFY ZD 2014008).

\section{Availability of data and materials}

The data that support the findings of this study are available from The Affiliated Hospital of Inner Mongolia Medical University but restrictions apply to the availability of these data, which were used under license for the current study, and so are not publicly available. Data are available from the 
authors upon reasonable request and with permission of The Affiliated Hospital of Inner Mongolia Medical University.

\section{Authors' contributions}

YW, LT and SX performed research and analyzed the data. YX, MZ and JZ contributed to the data collection and analysis. $\mathrm{ZW}$ contributed to the initial design of the project and the manuscript preparation. All the authors have approved the final version of the manuscript.

\section{Ethics approval and consent to participate}

Not applicable.

\section{Consent for publication}

Not applicable.

\section{Competing interests}

The authors declare that they have no competing interests.

\section{References}

1. McGlynn KA, Petrick JL and London WT: Global epidemiology of hepatocellular carcinoma: An emphasis on demographic and regional variability. Clin Liver Dis 19: 223-238, 2015.

2. Zetter BR: Angiogenesis and tumor metastasis. Annu Rev Med 49: 407-424, 1998

3. Folkman J: Role of angiogenesis in tumor growth and metastasis. Semin Oncol 29 (6 Suppl 16): S15-S18, 2002.

4. Volpert OV, Dameron KM and Bouck N: Sequential development of an angiogenic phenotype by human fibroblasts progressing to tumorigenicity. Oncogene 14: 1495-1502, 1997.

5. Liberman J, Sartelet H, Flahaut M, Mühlethaler-Mottet A, Coulon A, Nyalendo C, Vassal G, Joseph JM and Gross N: Involvement of the CXCR7/CXCR4/CXCL12 axis in the malignant progression of human neuroblastoma. PLoS One 7: e43665, 2012.

6. Wang J, Shiozawa Y, Wang J, Wang Y, Jung Y, Pienta KJ Mehra R, Loberg R and Taichman RS: The role of CXCR7/ $\mathrm{RDC1}$ as a chemokine receptor for CXCL12/SDF-1 in prostate cancer. J Biol Chem 283: 4283-4294, 2008.

7. Maishi N, Ohga N, Hida Y, Akiyama K, Kitayama K, Osawa T, Onodera Y, Shinohara N, Nonomura K, Shindoh M and Hida K: CXCR7: a novel tumor endothelial marker in renal cell carcinoma. Pathol Int 62: 309-317, 2012.

8. Yamada K, Maishi N, Akiyama K, Towfik Alam M, Ohga N, Kawamoto T, Shindoh M, Takahashi N, Kamiyama T, Hida Y, et al: CXCL12-CXCR7 axis is important for tumor endothelial cell angiogenic property. Int J Cancer 137: 2825-2836, 2015.
9. Chen Y, Teng F, Wang G and Nie Z: Overexpression of CXCR7 induces angiogenic capacity of human hepatocellular carcinoma cells via the AKT signaling pathway. Oncol Rep 36: 2275-2281, 2016.

10. Hu C, Shen SQ, Cui ZH, Chen ZB and Li W: Effect of microRNA-1 on hepatocellular carcinoma tumor endothelial cells. World J Gastroenterol 21: 5884-5892, 2015.

11. Cao Z, Zheng L, Zhao J, Zhuang Q, Hong Z and Lin W: Anti-angiogenic effect of Livistona chinensis seed extract in vitro and in vivo. Oncol Lett 14: 7565-7570, 2017.

12. Wani N, Nasser MW, Ahirwar DK, Zhao H, Miao Z, Shilo K and Ganju RK: C-X-C motif chemokine 12/C-X-C chemokine receptor type 7 signaling regulates breast cancer growth and metastasis by modulating the tumor microenvironment. Breast Cancer Res 16: R54, 2014.

13. Hao M, Zheng J, Hou K, Wang J, Chen X, Lu X, Bo J, Xu C, Shen K and Wang J: Role of chemokine receptor CXCR7 in bladder cancer progression. Biochem Pharmacol 84: 204-214, 2012.

14. Li F, Gao L, Jiang Q, Wang Z, Dong B, Yan T and Chen X: Radiation enhances the invasion abilities of pulmonary adenocarcinoma cells via STAT3. Mol Med Rep 7: 1883-1888, 2013.

15. Hida K, Maishi N, Torii C and Hida Y: Tumor angiogenesis-characteristics of tumor endothelial cells. Int J Clin Oncol 21: 206-212, 2016.

16. Müller A, Homey B, Soto H, Ge N, Catron D, Buchanan ME, McClanahan T, Murphy E, Yuan W, Wagner SN, et al: Involvement of chemokine receptors in breast cancer metastasis. Nature 410: 50-56, 2001.

17. Teicher BA and Fricker SP: CXCL12 (SDF-1)/CXCR4 pathway in cancer. Clin Cancer Res 16: 2927-2931, 2010.

18. Miao Z, Luker KE, Summers BC, Berahovich R, Bhojani MS, Rehemtulla A, Kleer CG, Essner JJ, Nasevicius A, Luker GD, et al: CXCR7 (RDC1) promotes breast and lung tumor growth in vivo and is expressed on tumor-associated vasculature. Proc Natl Acad Sci USA 104: 15735-15740, 2007.

19. Wu YC, Tang SJ, Sun GH and Sun KH: CXCR7 mediates TGF 31 -promoted EMT and tumor-initiating features in lung cancer. Oncogene 35: 2123-2132, 2016.

20. Yu H, Zhang L and Liu P: CXCR7 signaling induced epithelial-mesenchymal transition by AKT and ERK pathways in epithelial ovarian carcinomas. Tumour Biol 36: 1679-1683, 2015.

21. Lin L, Han MM, Wang F, Xu LL, Yu HX and Yang PY: CXCR7 stimulates MAPK signaling to regulate hepatocellular carcinoma progression. Cell Death Dis 5: e1488, 2014.

22. O'Shea JJ, Holland SM and Staudt LM: JAKs and STATs in immunity, immunodeficiency, and cancer. N Engl J Med 368: $161-170,2013$.

23. Xuan X, Li S, Lou X, Zheng X, Li Y, Wang F, Gao Y, Zhang H, $\mathrm{He} \mathrm{H}$ and Zeng Q: Stat3 promotes invasion of esophageal squamous cell carcinoma through up-regulation of MMP2. Mol Biol Rep 42: 907-915, 2015.

24. Zhao G, Zhu G, Huang Y, Zheng W, Hua J, Yang S, Zhuang J and Ye J: IL-6 mediates the signal pathway of JAK-STAT3-VEGF-C promoting growth, invasion and lymphangiogenesis in gastric cancer. Oncol Rep 35: 1787-1795, 2016.

25. Wang X, Qiu W, Zhang G, Xu S, Gao Q and Yang Z: MicroRNA-204 targets JAK2 in breast cancer and induces cell apoptosis through the STAT3/BCl-2/survivin pathway. Int J Clin Exp Pathol 8: 5017-5025, 2015. 